This work has been accepted for publication in JPO. Copyright may be transferred without further notice, and this version may no longer be accessible.

\title{
How Sensitive are Coarse General Circulation Models to Fundamental Approximations in the Equations of Motion?
}

\author{
Martin Losch, Alistair Adcroft, And Jean-Michel CAMpin \\ Department of Earth, Atmospheric, and Planetary Sciences, Massachusetts Institute of Technology, Cambridge, USA
}

\begin{abstract}
The advent of high precision gravity missions presents the opportunity to accurately measure variations in the distribution of mass in the ocean. Such a data source will prove valuable in state estimation and constraining general circulation models (GCMs) in general. However, conventional GCMs make the Boussinesq approximations, a consequence of which is that mass is not conserved. By use of the height-pressure coordinate isomorphism implemented in the MITgcm, the impact of non-Boussinesq effects can be evaluated. Although implementing a non-Boussinesq model in pressure coordinates is relatively straight-forward, making a direct comparison between height and pressure coordinate (i.e., Boussinesq and non-Boussinesq) models is not simple. But a careful comparison of the height coordinate and the pressure coordinate solutions ensures that only non-Boussinesq effects can be responsible for the observed differences. As a yard-stick, these differences are also compared to those between the Boussinesq hydrostatic and models in which the hydrostatic approximation has been relaxed, another approximation commonly made in GCMs. Model errors (differences) due to the Boussinesq and hydrostatic approximations are demonstrated to be of comparable magnitude. Differences induced by small changes in sub-grid scale parameterizations are at least as large. Therefore, non-Boussinesq and non-hydrostatic effects are most likely negligible with respect to other model uncertainties. However, because there is no additional cost incurred in using a pressure coordinate model, it is argued that non-Boussinesq modeling is preferable simply for tidiness. It is also concluded that even coarse resolution GCMs can be sensitive to small perturbations in the dynamical equations.
\end{abstract}

\section{Introduction}

Recently, the Boussinesq approximations in ocean models have attracted much attention (e.g., de Szoeke and Samelson 2002; Greatbatch et al. 2001; Huang and Jin 2002; Huang et al. 2001; Lu 2001; McDougall et al. 2002). The approximations, which are commonly employed for computational efficiency in general circulation models and in analytical studies, consist of replacing (i) mass conservation by volume conservation and (ii) the density in temporal and advection operators by a constant reference density (McDougall et al. 2002). ${ }^{1}$

While these approximations are generally justified for

Corresponding author address:

Martin Losch, Alfred-Wegener-Institut für Polar- und Meeresforschung, Postfach 120161, 27515 Bremerhaven, Germany, e-mail: mlosch@awi-bremerhaven.de

${ }^{1}$ Spiegel and Veronis (1960) summarized the Boussinesq approximations as follows: "(1) The fluctuations in density which appear with the advent of motion result principally from thermal (as opposed to pressure) effects. (2) In the equations for the rate of change of momentum and mass, density variations may be neglected except when they are coupled to the gravitational acceleration in the buoyancy force." purposes of simulating the ocean circulation with ocean general circulation models (OGCMs), there are many problems in physical oceanography that may require the use of nonBoussinesq OGCMs. Boussinesq models conserve volume; consequently, they cannot recover steric effects. Hence, unless the steric sea level change is explicitly calculated (Greatbatch 1994), one cannot use such models to study global sea level change due to net heating of the ocean at seasonal and longer time-scales. Furthermore, changes in the heat and freshwater content of the ocean can have spurious effects on the diagnosed bottom pressure in OGCMs that make the Boussinesq approximations. For example, heating the water column (and neglecting the subsequent adjustment) decreases the density. By volume conservation, decreasing the density reduces the mass and the bottom pressure, which for a real fluid should be unchanged in this case. Therefore, a volume conserving model may be inappropriate to study oceanic mass distribution and bottom pressure in the context of high precision satellite gravity missions such as the GRACE (Gravity Recovery and Climate Experiment) mission (Greatbatch et al. 2001). Reducing the mass by heating from above also has dynamical consequences that can re- 
sult in different adjustment processes and different sea surface elevations of a Boussinesq and a non-Boussinesq model (Huang and Jin 2002).

According to Huang et al. (2001), the Boussinesq approximations also may introduce erroneous energy sources and energy transformation processes, although this issue is under debate (R. Ferrari and A. Adcroft, personal correspondence). In the ocean, heating the water column from above raises the sea surface and increases the gravitational potential energy. But in a Boussinesq model, the same heating decreases the mass, does not raise the sea level, and thus reduces the gravitational potential energy. Also, neglecting the compressibility in the continuity equation removes the explicit conversion between mechanical and internal energy from the Boussinesq model. This may have an effect on the energy balance in the Boussinesq equations, although the magnitude of these errors is unclear (Huang et al. 2001).

In this paper, we will argue that all of these errors are at the noise level of a coarse resolution OGCM. In particular, they are comparable to, say, errors due to the hydrostatic approximation and uncertainties associated with model parameterizations, because the long integration of an OGCM is sensitive to any small perturbation, even at non-eddypermitting resolution.

McDougall et al. (2002) pointed out that—in addition to replacing mass conservation by volume conservation and the density by a constant reference density in temporal and advection operators-when making the Boussinesq approximations, an error in the tracer equation needs to be considered that results from using a divergence-free velocity as the advecting velocity. Greatbatch et al. (2001) and Lu (2001) each suggested a practical solution for accounting for that error in a conventional Boussinesq model. The two solutions differ only slightly: Lu (2001) re-interpreted the model variables as density weighted averages of the grid cell and added a correction to the vertical advection term in the momentum and tracer balance equations. This correction term is diagnosed from the original continuity equation which includes time-derivatives in density. Greatbatch et al. (2001) re-interpreted the model velocity variables as average mass flux per area normalized by a constant reference density. They arrived at a set of equations in which the density needs to be stepped forward in time.

In a completely different approach, de Szoeke and Samelson (2002) showed that the non-Boussinesq hydrostatic equations in pressure coordinates have a form that is "dual" to the hydrostatic Boussinesq equations. In this way, only the structure of the boundary conditions in existing Boussinesq ocean model code needs to be modified. The remaining code can be used without any further modifications, provided one replaces depth, vertical velocity, pressure, and scaled density anomaly by pressure, vertical pseudo-velocity, Montgomery potential, and the scaled specific volume anomaly, respectively.

In the MITgcm (Marshall et al. 1997a, the model code is available at http://mitgcm.org), the dynamical core of the model suggested by de Szoeke and Samelson (2002) is already implemented and used as an atmospheric model (see Marshall et al. 2003, where atmosphere-ocean isomorphisms and their implementation in the MITgcm are described). In fact, the MITgcm was originally motivated by an atmospheric model in pressure coordinates (Brugge et al. 1991). By transferring the atmospheric model to the ocean and replacing the equation of state, a fully non-Boussinesq OGCM in pressure coordinates is readily available. Here, this model is integrated in parallel with the Boussinesq height coordinate mode of the MITgcm and the solutions are compared to yield a quantitative assessment of the differences due to the Boussinesq approximation. The MITgcm can also be run as a non-hydrostatic model which makes it possible to compare the relative impact of the Boussinesq and the hydrostatic approximations and check the conclusion of de Szoeke and Samelson (2002) that the crucial simplification is the latter one.

\section{Non-Boussinesq Pressure Coordinate Model and Boussinesq Height Coordinate Model: Making the Models Comparable}

Following de Szoeke and Samelson (2002), the hydrostatic, Boussinesq equations of motion in height coordinates have the same form as the hydrostatic, non-Boussinesq equation in pressure coordinates. To obtain a set of nonBoussinesq equations in pressure coordinates from Boussinesq equations in height coordinates, one only has to substitute pressure for height as the vertical coordinate, a pseudovelocity, which is the rate of change of pressure, for the vertical velocity, the geopotential height for hydrostatic pressure and specific volume for density. Marshall et al. (2003) described how this isomorphism of the equations is exploited for modeling of the atmosphere and the ocean with the same dynamical kernel code. The description of the isomorphism and its application to a non-Boussinesq pressure coordinate model of the ocean are summarized in Appendix A.

In the following sections, the solution of the nonBoussinesq pressure coordinate model is compared to that of the Boussinesq height coordinate model for a coarse resolution configuration with mixed boundary conditions. Although both models use essentially the same dynamical kernel of the MITgcm, details of the implementation and the parameters of the two models render the direct comparison of the two model difficult. Before the models can be compared, the following issues need to be addressed.

\section{a. Initialization}

Because the vertical grids of the pressure coordinate and height coordinate models are different, the models cannot be restarted from a common spun-up equilibrium state. Only a start from rest with flat hydrography provides the identical initial conditions that are necessary for the close comparison presented here. Difficulties in interpolating the hydrography onto pressure levels that implicitly depend on temper- 
ature and salinity are avoided by starting both models from uniform temperature and salinity fields, where the constant values of $\theta=3.6^{\circ} \mathrm{C}$ and $S=34.7$ are chosen to be approximately the mean temperature and salinity estimated from the Levitus climatologies (Levitus and Boyer 1994; Levitus et al. 1994).

Pressure is a nonlinear function of depth. Therefore, after choosing the depth levels in the height coordinate model, the pressure levels in the non-Boussinesq model and the initial pressure field in the Boussinesq model are determined by integrating the nonlinear hydrostatic equation $d p=-g \rho(p) d z$. In the Boussinesq model, the pressure is evaluated at the end of a time step. Then at the beginning of the next time step, the pressure from the previous time step is used in the equation of state to calculate density. Lagging pressure in this way avoids the nonlinear integration at every time step (Griffies et al. 2001). Using an equation of state in which pressure is computed as $p(z)=-g \rho_{0} z$ can lead to errors of up to a few Sverdrups (1 Sverdrup $\left.=1 \mathrm{~Sv}=10^{6} \mathrm{~m}^{3} \mathrm{~s}^{-1}\right)$ in the Gulf Stream region (Dewar et al. 1998) and is therefore explicitly avoided in our comparison. Huang and Jin (2002) used an equation of state that does depend on height and not on pressure in the height coordinate model which makes definite comparisons between Boussinesq and non-Boussinesq models problematic. In this study, density is computed as a function of pressure in both height coordinate and pressure coordinate model.

\section{b. Computation of the Potential}

The potential $\phi$ ( $\phi$ is pressure divided by $\rho_{0}$ in height coordinates and geopotential height in pressure coordinates) is computed by integrating the generalized buoyancy $b$ starting from the free surface $r_{s}$ ( $b$ is gravitational acceleration times density in height coordinates and specific volume in pressure coordinates; see Appendix A for a further explanation of the notation):

$$
\phi(r)=\phi\left(r_{s}\right)+\int_{r_{s}}^{r} b\left(r^{\prime}\right) d r^{\prime},
$$

where $r$ is the general vertical coordinate.

At rest and with no atmospheric pressure load, the sea surface of a homogeneous ocean is flat. Because both pressure and geopotential are zero at the air-sea interface, this implies that, in this resting state, the potential $\phi$ must be zero at the ocean-atmosphere interface in both formulations. This condition is easily met in the height coordinate formulation where the air-sea interface is the free surface and $\phi\left(r_{s}\right)=p(z=\eta) / \rho_{0} \equiv 0$. In the pressure coordinate model, on the other hand, the "free surface" $r_{s}$ is at the ocean floor and appropriate boundary values $\phi\left(r_{s}\right)$ at the bottom are needed to ensure that, at rest, the geopotential is flat at the air-sea interface. These boundary values are obtained by integrating the initial $b$ from the free surface at rest $r_{s}^{0}=r_{s}(t=0)$ to the fixed surface $r=R_{\text {fixed }}(=0 \mathrm{~Pa})$ at the ocean-atmosphere interface:

$$
\phi\left(r_{s}\right)=-\left.\int_{r_{s}^{0}}^{R_{\mathrm{fixed}}} b\left(r^{\prime}\right)\right|_{t=0} d r^{\prime}
$$

\section{c. Natural Boundary Conditions for Freshwater Flux}

The response to freshwater forcing is anticipated to be one of the major dynamical differences between a Boussinesq and a non-Boussinesq model (Huang and Jin 2002). Hence, the implementation of the natural boundary conditions for freshwater flux requires great care.

In the height coordinate model, adding freshwater locally reduces the salinity of the top layer, but at the same time increases the height of the free surface via an inhomogeneous term in the free surface equation (Equation (A9) in Appendix A). Note, that in this work, the freshwater flux is balanced globally over the forcing period (one year) so that there is no net flux of freshwater into the ocean.

In pressure coordinates, the flux of freshwater is a mass flux at the surface, also balanced to avoid an overall mass drift. The surface mass fluctuations $g \rho_{F W}(P-E)$ change the pressure tendency $\omega=D p / D t$ by a vertically constant value throughout the entire water column. Consequently, $g \rho_{F W}(P-E)$ also appears as a forcing term in the bottom pressure equation(A20) in Appendix A, which is effectively a mass equation. The salinity, however, is affected in the surface layer. The derivation of the surface boundary conditions for $\omega$ in pressure coordinates can be found in Appendix B.

\section{d. Free Surface vs. Bottom Pressure Gradients in the Momentum Equations}

In the height coordinate model the contribution to the momentum equations of the surface pressure gradient is split into $g \nabla \eta+g \nabla\left[\left(\rho-\rho_{0}\right) / \rho_{0}\right] \eta$. Often, the second term is neglected on the grounds that $\left(\rho-\rho_{0}\right) / \rho_{0} \ll 1$. But in pressure coordinates and with topography, the corresponding geopotential height gradient at the bottom is evaluated at different pressures. Making the above approximation in pressure coordinates introduces larger errors because the specific volume varies with depth. Therefore, terms of order $\left(\rho-\rho_{0}\right) / \rho_{0}$ in height coordinates and $\left(\alpha-\alpha_{0}\right) / \alpha_{0}$ in pressure coordinates $\left(\alpha=\rho^{-1}\right)$ are not neglected in this study, thereby reducing the differences between a height coordinate and a pressure coordinate model.

\section{e. Turbulent Diffusion and Viscosity}

The spatially constant eddy diffusion and eddy viscosity coefficients of the height coordinate (Boussinesq) model have to be converted to pressure coordinates. For example, the vertical viscosity terms takes the form

$$
\frac{\partial}{\partial z}\left(A_{V}^{(z)} \frac{\partial \mathbf{u}}{\partial z}\right) \mapsto g^{2} \rho \frac{\partial}{\partial p}\left(A_{V}^{(z)} \rho \frac{\partial \mathbf{u}}{\partial p}\right)
$$


where $A_{V}^{(z)}$ is the vertical eddy viscosity coefficient, $\mathbf{u}$ the horizontal velocity vector, $g$ the acceleration due to gravity, and $\rho$ the in-situ density. Therefore only for constant density $\rho=\rho_{0}$, does $A_{V}^{(p)}=A_{V}^{(z)} g^{2} \rho_{0}^{2}$. However, for any realistic scenario, in which pressure is a nonlinear function of density, the vertical diffusion and viscosity coefficients are a function of the vertical coordinate. Here, these variations are neglected and the coefficients $A_{V}^{(p)}$ and $\kappa_{V}^{(p)}$ are assumed constant. This assumption introduces an error of up to $3 \%$ in the vertical viscosity and diffusion terms, if one assumes a reference density of $\rho_{0}=1035 \mathrm{~kg} \mathrm{~m}^{-3}$. Thus, the vertical eddy viscosity and diffusivity coefficients should be scaled by $\left(\rho / \rho_{0}\right)^{2}$ to reduce this error but are not done so here. There is a further discrepancy in the lateral diffusion and viscosity terms; in the height coordinate model lateral eddy fluxes are directed down-gradient along the horizontal. In pressure coordinates the fluxes are calculated along isobaric surfaces which may be inclined. But the slope of isobaric surfaces is at most of the order $10^{-4}$ and this effect is neglected as well. Finally, implementing the Gent-McWilliams and Redi schemes (Gent and McWilliams 1990) in pressure coordinates would have incurred the same difficulties and so we chose to not employ these schemes.

\section{Comparison of OGCM Results}

\section{a. Model Parameters and Configuration}

Both models are integrated for 1000 years. The horizontal resolution is $4^{\circ}$, ranging from $80^{\circ} \mathrm{S}$ to $80^{\circ} \mathrm{N}$ with 15 vertical levels. The bottom topography is realistic and derived from ETOPO5 (NOAA 1988). The level thickness ranges from $50 \mathrm{~m}$ to $690 \mathrm{~m}$ in the height coordinate model. Monthly mean wind stress fields by Trenberth et al. (1990), monthly mean heat flux and climatological freshwater flux by Jiang et al. (1999) force the models at the surface. The surface layer of thickness $50 \mathrm{~m}$ is also restored with a time scale of 2 months to monthly mean sea surface temperature (Levitus et al. 1994) to represent an oceanic feedback on the actual heat flux. Basic parameters of both models are summarized in Table 1. The comparison between the pressure coordinate and height coordinate model is carried out by showing the differences of time averaged fields. Unless indicated otherwise, the averages are taken over the last 100 years of the integration.

The two models operate on different vertical grids. Hence, direct comparison of model variables will in most cases involve the interpolation from one grid to the other, introducing another possible, albeit small, source of differences between the results. The only variables that evade this problem are bottom pressure and sea surface elevation. Bottom pressure is a prognostic variable in the pressure coordinate model, but must be diagnosed from the height coordinate model. In contrast, sea surface elevation has to be diagnosed in the pressure coordinate model, but is a prognostic variable in the height coordinate model. Furthermore, the mean bottom pressure in the height coordinate model may

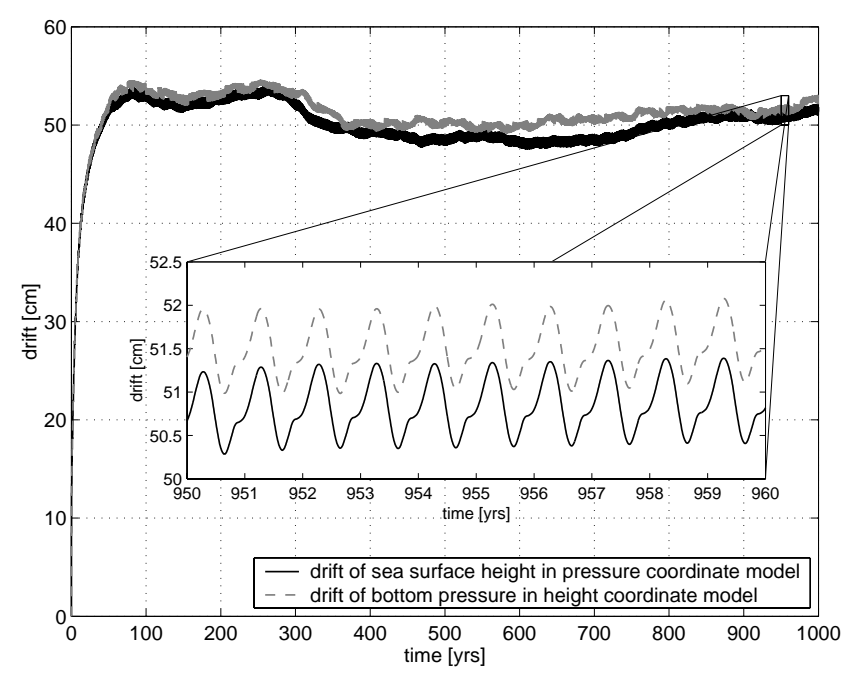

FIG. 1. Temporal evolution of the global means of bottom pressure of the volume conserving (height coordinate, Boussinesq) model and sea surface height of the mass conserving (pressure coordinate, non-Boussinesq) model. The bottom pressure is represented in height units after scaling by $g \rho_{0} \approx 10^{4} \mathrm{~kg} \mathrm{~m}^{-2} \mathrm{~s}^{-2}$ and reversing the sign. Both models show both an annual cycle and variability of longer time scales.

contain a false mass drift in time, whereas the mean sea surface elevation in the pressure coordinate model may drift due to steric expansion. ${ }^{2}$ Fig. 1 shows the time evolution of the globally averaged bottom pressure of the height coordinate model and the globally averaged sea surface height of the pressure coordinate model. The evolution of the global mean of bottom pressure in the height coordinate model in Fig. 1 is represented in height units after scaling by $g \rho_{0}$ and reversing the sign. Note that in spite of the approximate scaling (one could use the vertically averaged in situ-density instead of $\rho_{0}$ ), the mass evolution in the height coordinate model and the volume evolution in the pressure coordinate model are remarkably similar. We thereby confirm the conclusion of Greatbatch (1994) who showed that one can recover steric effects by adjusting the sea level by a globally uniform, timevarying correction.

\section{b. What is the Magnitude of the Differences?}

We now compare sea surface elevations and bottom pressure anomalies (differences from the long time mean). For this comparison, both the time-dependent global averages of the total mass of the height coordinate model and the volume of the pressure coordinate model have been removed.

To gauge how important the observed differences between the Boussinesq and the non-Boussinesq model are, we compare the height coordinate model with the same model

\footnotetext{
${ }^{2}$ Diagnosing bottom pressure in the height coordinate model or surface elevation in the pressure coordinate model is only consistent with the model discretization if the hydrostatic equation is integrated using a finite volume discretization as opposed to finite difference discretization. The finite volume discretization of the hydrostatic equation is described in Appendix C.
} 
Table 1. Summary of model parameters

\begin{tabular}{llrr}
\hline \multicolumn{1}{c}{ Parameter } & Symbol & \multicolumn{1}{c}{$z$-coordinates } & \multicolumn{1}{c}{$p$-coordinates } \\
\hline horizontal viscosity & $A_{H}$ & $3.00 \times 10^{+5} \mathrm{~m}^{2} \mathrm{~s}^{-1}$ & $3 \times 10^{5} \mathrm{~m}^{2} \mathrm{~s}^{-1}$ \\
vertical viscosity & $A_{V}$ & $1.67 \times 10^{-3} \mathrm{~m}^{2} \mathrm{~s}^{-1}$ & $1.721611620915750 \times 10^{5} \mathrm{~Pa}^{2} \mathrm{~s}^{-1}$ \\
horizontal diffusivity & $\kappa_{H}$ & $1.00 \times 10^{+3} \mathrm{~m}^{2} \mathrm{~s}^{-1}$ & $1 \times 10^{3} \mathrm{~m}^{2} \mathrm{~s}^{-1}$ \\
vertical diffusivity & $\kappa_{V}$ & $5.00 \times 10^{-5} \mathrm{~m}^{2} \mathrm{~s}^{-1}$ & $5.154525811125000 \times 10^{3} \mathrm{~Pa}^{2} \mathrm{~s}^{-1}$ \\
convective vertical diffusivity & $\kappa_{\text {impl }}$ & $1.00 \times 10^{+1} \mathrm{~m}^{2} \mathrm{~s}^{-1}$ & $1.030905162225000 \times 10^{9} \mathrm{~Pa}^{2} \mathrm{~s}^{-1}$ \\
bottom friction & $r$ & 0 & 0 \\
reference density & $\rho_{0}$ & $1035.0 \mathrm{~kg} \mathrm{~m}^{-3}$ & $1035.0 \mathrm{~kg} \mathrm{~m}^{-3}$ \\
freshwater reference density & $\rho_{F W}$ & $999.8 \mathrm{~kg} \mathrm{~m}^{-3}$ & $999.8 \mathrm{~kg} \mathrm{~m}^{-3}$ \\
\hline
\end{tabular}

with small changes to the dynamics, the parameterizations, and the forcing fields. The most prominent change is the relaxation of the hydrostatic approximation to include the vertical Coriolis terms and non-hydrostatic metric terms that include the vertical velocity $w$. (See Appendix D for details.) With these terms, which are generally of the order of $10^{-5} \mathrm{~m}^{2} \mathrm{~s}^{-1}$, the model is not fully non-hydrostatic, but it has a consistent energy conservation principle. Marshall et al. (1997b) called this model "quasi-hydrostatic".

We also compare two hydrostatic Boussinesq models that differ only in the choice of the vertical diffusivity parameter for temperature and salinity. This parameter is often tuned to bring the model close to the observations. Therefore it can have values that vary dramatically from one application to another. As an additional test, we compare the results of the height-coordinate model with different implementations of the equation of state and perturbations of the forcing fields at the level of numerical round-off errors.

The mean sea surface of the Boussinesq model is shown in the top panel of Fig. 2. The difference in mean sea surface elevation between the Boussinesq and non-Boussinesq models in the second panel of Fig. 2 reaches $4 \mathrm{~cm}$ in the Southern Ocean, otherwise it is small. Such differences will just be detectable when the new high precision geoid models become available which are expected from the ongoing gravity mission GRACE. The third panel of Fig. 2 shows the difference in mean sea surface height due to quasi-hydrostatic terms. This effect is approximately half the Boussinesq effects. The bottom panel of Fig. 2 compares the Boussinesq model to an experiment in which the vertical diffusivity has been increased by $1 \%$ of the standard value. Apparently, this change in diffusivity leads to similar, if not greater, changes in mean sea surface elevation as do the Boussinesq effects.

In general, the differences due to Boussinesq, hydrostatic, or equation-of-state effects are largest in the Southern Ocean. This region is characterized by large horizontal density gradients and steep isopycnal slopes. Small changes in these large gradients may explain the observed sensitivity to small perturbations. Since these calculations do not have the Gent-McWilliams parameterization (GM), there is more convective activity in the Southern Ocean (ACC) than is normally seen in models with GM. The highly non-linear nature of the convection parameterization may also be contributing to the sensitivity of the Southern Ocean in our results.

The TOPEX/POSEIDON altimeter mission provided oceanographers with sea surface height anomaly data that have an accuracy of the order of $2 \mathrm{~cm}$ (Wunsch and Stammer 1998). The accuracy of these data may serve as the benchmark for the difference in sea surface variability. The square root of the variance of the sea surface height over 100 model years is shown in the top panel of Fig. 3 for the Boussinesq model. The difference in sea surface height variability between Boussinesq and non-Boussinesq model (second panel of Fig. 3) is smaller than that due to quasi-hydrostatic terms (third panel of Fig. 3). It is hardly detectable with today's high precision altimetry.

All experiments so far use the polynomial equation of state derived by Jackett and McDougall (1995) from the UNESCO formula. The bottom panel of Fig. 3 shows the difference due to a different equation of state, namely the polynomial published by McDougall et al. (2003). While the difference in the density computed by these different formulas is of the order of $10^{-3} \mathrm{~kg} \mathrm{~m}^{-3}$, it still causes differences in sea surface variability of the same order as those due to Boussinesq effects.

Bottom pressure gauges and the satellite mission GRACE produce measurements of the bottom pressure fluctuations. We therefore compare the temporal variations of bottom pressure in the models. The top panel of Fig. 4 shows the square root of the bottom pressure variance over a period of 100 years for the Boussinesq model (with the global mean subtracted at every time step). The difference in the bottom pressure variability between the Boussinesq model and the non-Boussinesq model (second panel of Fig.4) is on the order of $1 \mathrm{~cm}$ equivalent sea surface height in the Southern Ocean and much smaller everywhere else. This difference is as large as $30 \%$ of the signal and is therefore not negligible. However, the difference in bottom pressure variability due to the quasi-hydrostatic effects (third panel of Fig.4) has a still larger amplitude. 

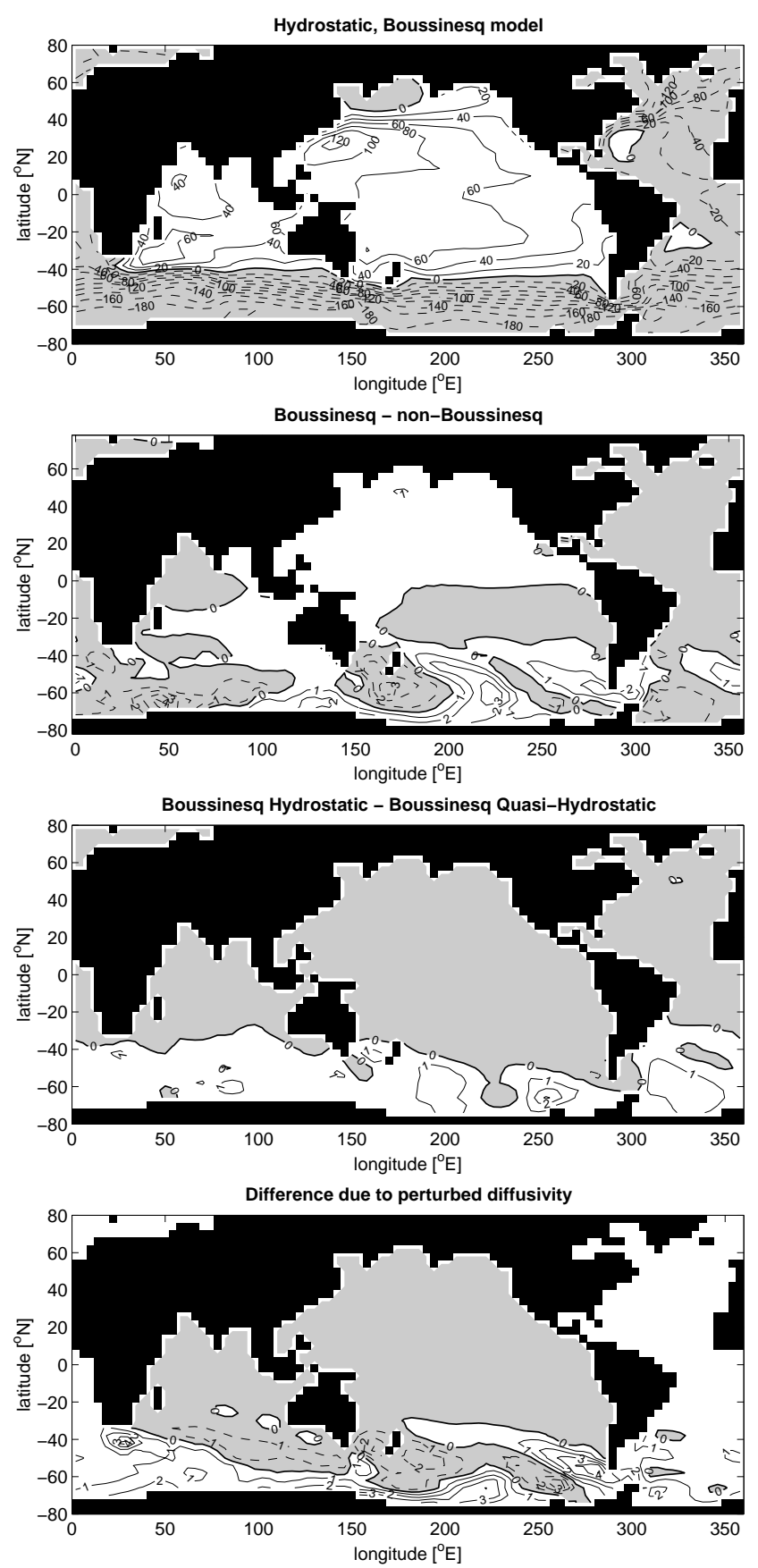

FIG. 2. Top panel: mean sea surface elevation (in $\mathrm{cm}$ ) of the hydrostatic, Boussinesq model; contour interval is $20 \mathrm{~cm}$. Second panel: sea surface height difference due to Boussinesq effects; contour interval is $1 \mathrm{~cm}$. Third panel: sea surface height differences due to quasi-hydrostatic effects; contour interval is $1 \mathrm{~cm}$. Bottom panel: difference in sea surface height variability due to a change of $1 \%$ in vertical diffusivity; contour interval is $1 \mathrm{~cm}$.

To assess the extent to which the above responses are an innate property of the system (i.e., does any small perturbation lead to large changes?), the height coordinate model run is repeated with slightly different forcing fields: all forcing fields are perturbed by random noise with a relative amplitude of $2.22 \times 10^{-16}$. This amounts to changing the last
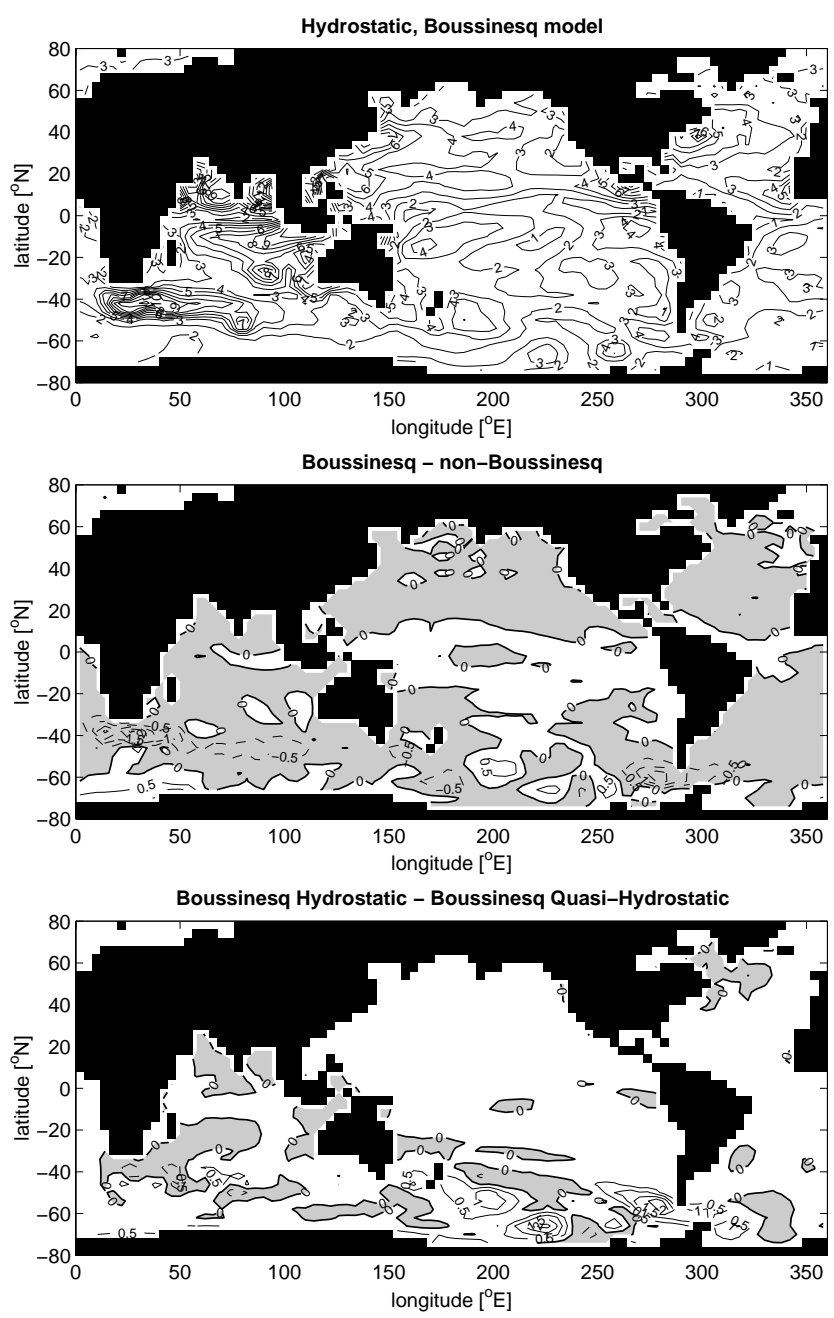

Difference due to changed EOS

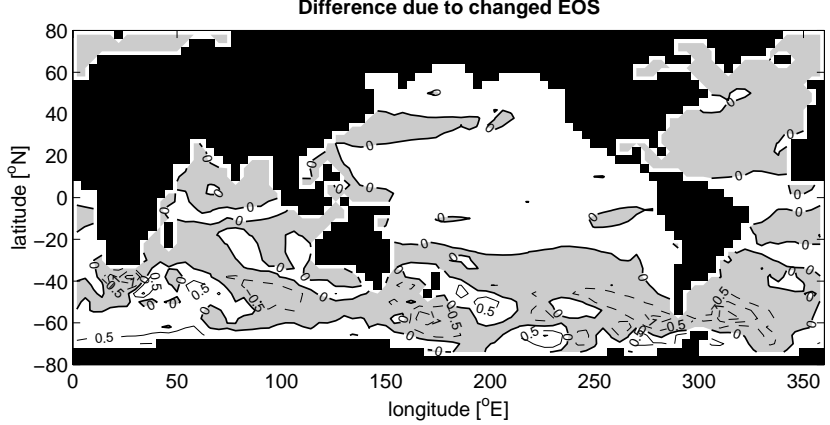

FIG. 3. Top panel: Sea surface height variability (square-root of the variance in $\mathrm{m}$ ); contour interval is $1 \mathrm{~cm}$. Second panel: difference in sea surface height variability due to Boussinesq effects; contour interval is $0.5 \mathrm{~cm}$. Third panel: difference in sea surface height variability due to quasi-hydrostatic effects; contour interval is $0.5 \mathrm{~cm}$. Bottom panel: sea surface height differences due to a changed equation of state; contour interval is $0.5 \mathrm{~cm}$.

digit of a 64 bit (double precision) real number and simulates the differences in round-off error that one encounters when changing compilers or computing platforms. The bottom panel of Fig. 4 shows the resulting differences in bottom pressure variability. As with the use of a different equation of state and a perturbed vertical diffusivity, the effect of 

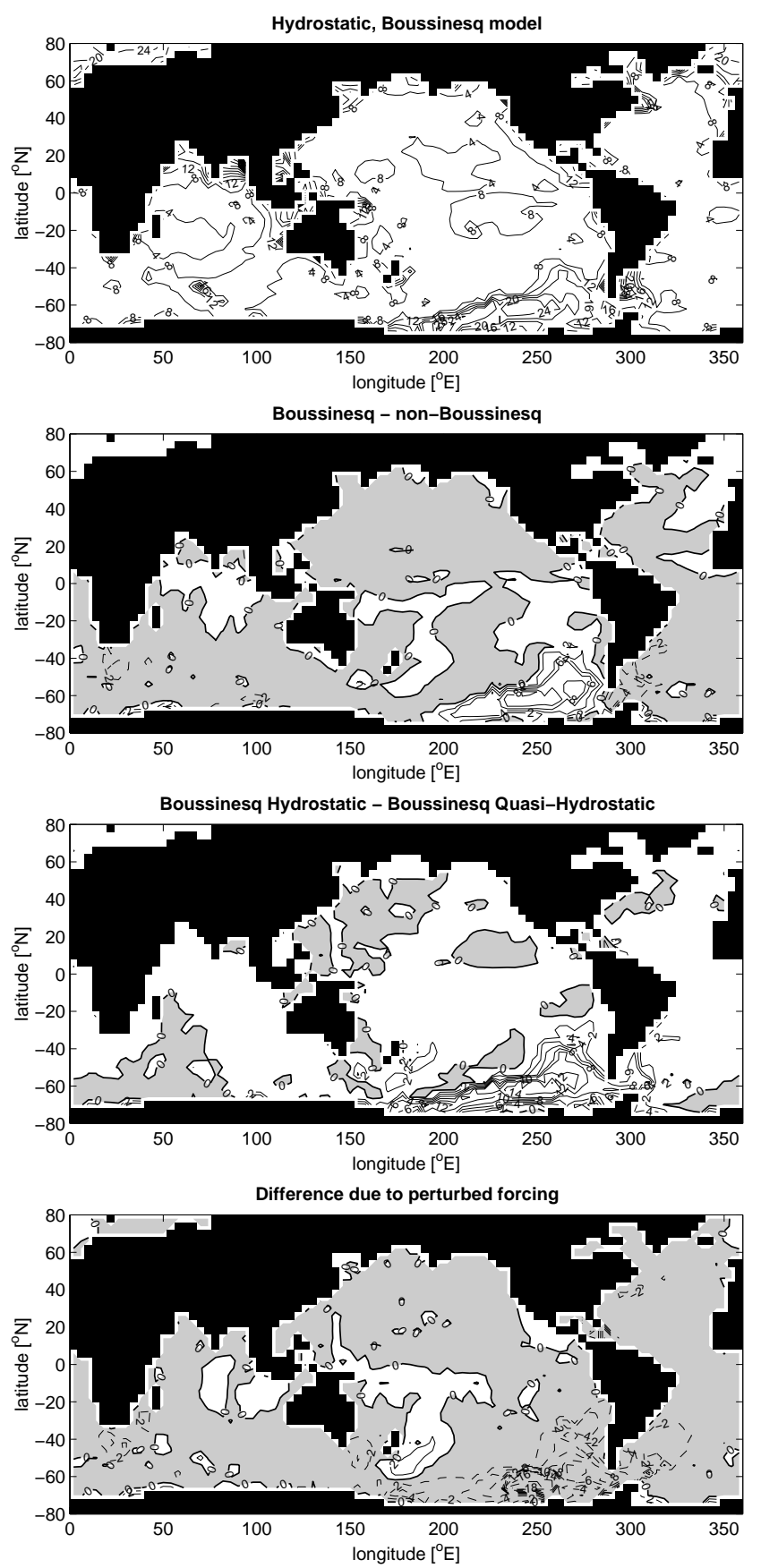

FIG. 4. Top panel: bottom pressure variability (square root of the variance); contour interval is $40 \mathrm{~Pa} \approx 4 \mathrm{~mm}$ equivalent sea surface height. Second panel: difference in bottom pressure variability due to Boussinesq effects; contour interval is $10 \mathrm{~Pa} \approx 1 \mathrm{~mm}$. Third panel: difference in bottom pressure variability due to quasihydrostatic effects; contour interval is $10 \mathrm{~Pa} \approx 1 \mathrm{~mm}$. Bottom panel: difference in bottom pressure variability due to numerical noise in the forcing fields; contour interval is $10 \mathrm{~Pa} \approx 1 \mathrm{~mm}$.

these tiny perturbations on the variability is of the same order of magnitude as the Boussinesq effects. This again suggests, that our model is particularly sensitive in the Southern Ocean, where the flow is geostrophically balanced by large density gradients.

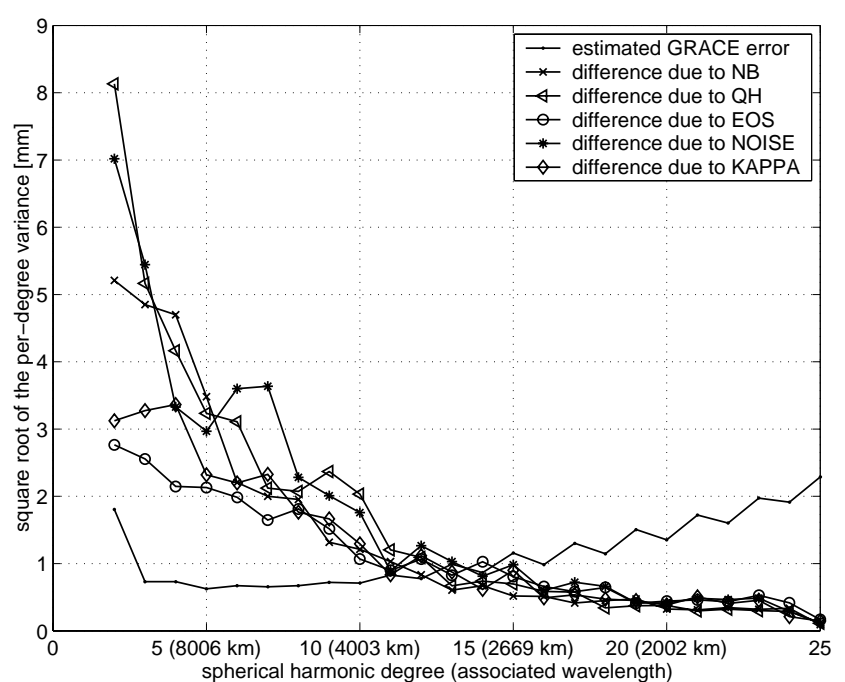

FIG. 5. The difference in bottom pressure variability as a function of scale. Shown is the square root of the degree variances $\sqrt{\sum_{m}\left|c_{n m}\right|^{2}}$ of the spherical harmonic coefficients $c_{n m}$. All approximations and errors give rise to differences in bottom pressure variability that exceed the estimated errors of a geoid derived from GRACE (Balmino et al. 1998) at large scales. But the hydrostatic approximation $(\mathrm{QH})$, small changes in the vertical diffusivity (KAPPA), small differences in the equation of state (EOS), and numerical noise in the forcing fields (NOISE) seem to be as important as Boussinesq effects (NB).

The most likely explanation for the observed sensitivity is the choice of mixed boundary conditions. The combination of restoring conditions for temperature and flux boundary conditions for salinity has been reported to make OGCMs unstable (Power and Kleeman 1994; Rahmstorf 1996). Repeating the above experiments with restoring boundary conditions for both temperature and salinity constrains the height coordinate and the pressure coordinate model to stay on very similar trajectories. However, this does not change the qualitative result: when the models are restored to the same surface fields, the solutions of the various models become more similar and all differences are reduced by one order of magnitude. But the relative sizes of the differences remain the same (not shown).

Fig. 5 summarizes the previous comparisons as a function of scale. Plotted is the square-root of the degree variances $\sqrt{\sum_{m}\left|c_{n m}\right|^{2}}$ of the bottom pressure variability differences (in $\mathrm{mm}$ equivalent sea surface height) projected onto spherical harmonic coefficients $c_{n m}$. To bring the experiments into the context of the current high precision satellite gravity mission GRACE, the expected error of a geoid model that will be derived from the GRACE gravity data (Balmino et al. 1998 ) is also drawn as a function of spherical harmonic degree. (It is assumed that the bottom pressure measurement errors, which are unknown at this time, are comparable to the errors of the mean geoid. In this sense, the geoid errors provide only a rough estimate of the measurement errors.) All differences in bottom pressure variability are larger than 
the geoid errors on the very large scales; degree 10 corresponds to a wavelength of approximately $4000 \mathrm{~km}$, degree 15 to approximately $2670 \mathrm{~km}$. On shorter scales the geoid errors dominate. For most coefficients, that is spatial scales, the differences due to Boussinesq effects are smaller than those due to both non-hydrostatic effects and round-off noise in the forcing fields.

In summary, comparison of solutions obtained using Boussinesq and non-Boussinesq models shows significant differences in the time-mean and variance of surface elevation and in the variance of bottom pressure. Comparison of the hydrostatic model to the quasi-hydrostatic model indicates larger differences than those due to the non-Boussinesq approximations in sea-surface height and bottom-pressure variances though a considerably smaller change in mean sea surface height. Thus the relative effect of the two approximations seems comparable. Changing the vertical diffusivity by $1 \%$ - actually, we know that both vertical viscosity and diffusion can potentially vary by $2-3$ orders of magnitudethe form of the equation of state, or adding truncation level noise to the forcing leads to equally large changes.

\section{Are the Differences Between Height Coordinate and Pressure Coordinate Model Really due to the Boussinesq Approximations?}

After showing that the differences between the Boussinesq and the non-Boussinesq model are generally of the same order of magnitude as those due to relaxing the hydrostatic approximation or changing uncertain parameters slightly, it is still not clear whether the observed differences between the models are really due to the Boussinesq approximation or simply due to the numerical difference introduced by the different coordinate systems. These may arise because in a physically identical fluid the gradient operators act along different surfaces (pressure vs. height surfaces), so that numerical truncation may lead to different trajectories. The nonlinear free surface causes an additional numerical difference: in the height coordinate model the surface layer has a variable thickness, while in the pressure coordinate model it is the bottom layer that can vary in time and space (Campin et al. 2003).

To test how much of the differences between the height coordinate model and the pressure coordinate model are due to the numerical discretization, any dependence on the Boussinesq approximations is removed by replacing the pressure dependent density with a constant in both the height coordinate model and the pressure coordinate model. The system is forced by wind stress only, and there is no buoyancy flux. Then any pressure level inclination is solely barotropic and due to sea surface elevation changes.

Only two differences remain between the pressure coordinate and the height coordinate model: changes in layer thickness due to the nonlinear free surface (at the bottom in pressure coordinates and at the top in the height coordinate model) and the way the horizontal gradients in the nonlinear
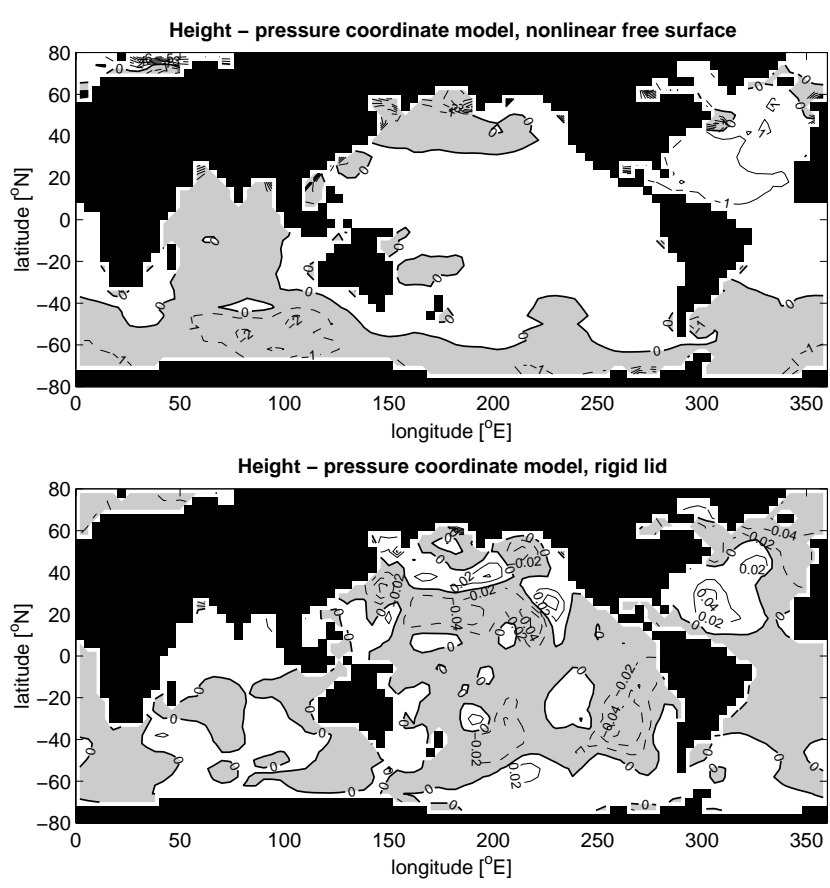

FIG. 6. Difference of the square root of the sea surface variance over an averaging period of 1 year for the model with constant density. Top panel: with nonlinear free surface; contour interval is $1 \times 10^{-6} \mathrm{~m}$. Bottom panel: with rigid lid; contour interval is $0.02 \times 10^{-6} \mathrm{~m}$.

advection terms and the horizontal viscosity terms are evaluated (strictly along pressure surfaces and along horizontal surfaces, which form a small angle with the pressure surfaces). Note that in this configuration, $A_{V}^{(p)} \equiv A_{V}^{(z)} g^{2} \rho_{0}^{2}$, exactly.

Fig. 6 illustrates the contributions of the effects due to the nonlinear free surface. Shown is the difference in sea surface height variability. Because of its barotropic nature the model equilibrates very quickly and the averaging period is the 10th year of integration. Although both the height coordinate and the pressure coordinate model describe the same fluid of constant density, the answers are slightly different due to the different truncation errors implied by the different formulation, top panel of Fig. 6. The difference between pressure and height coordinate model is three orders of magnitude smaller than in the full model of Section 3. Replacing the nonlinear free surface by a rigid lid, bottom panel of Fig.6, decreases the difference in sea surface height variability further by two orders of magnitude (and the differences in mean sea surface elevation and bottom pressure variability as well, not shown).

The remaining differences in sea surface height variability are largely due to the fact, that sea surface elevation in the pressure coordinate model is a diagnostic variable, whereas it is prognostic in the height coordinate model. In a barotropic model, sea surface height and bottom pressure are equivalent. Comparing the sea surface height of the height coordinate model and the bottom pressure anomaly of the 
pressure coordinate model reveals that, after proper scaling, they are the same in the case of a rigid lid to working precision. The differences in horizontal velocities are also smaller than $10^{-14}$ for the barotropic experiment with rigid lid, and the two models give essentially the same result. That is, the angles between surfaces of constant height and surfaces of constant pressure are so small that the horizontal gradients do not cause any differences that exceed the noise level. We can assume that in the case of variable density, the differences in numerical discretization are also mainly due to the nonlinear free surface. Although there may be a larger impact of the free surface in the full baroclinic model because of the vertical advection of buoyancy near the surface, the numerical effects due to different coordinate systems are still much smaller than the Boussinesq effects.

\section{Discussion and Conclusions}

Conventional OGCMs make a number of approximations that influence their solution, such as the hydrostatic approximation and the Boussinesq approximations. We find that relaxing the hydrostatic approximation has a larger impact on the variability of a coarse resolution global model than do Boussinesq effects. Non-Boussinesq effects lead to larger changes in mean sea surface elevation than do non-hydrostatic effects and this is consistent with switching from conserving volume to conserving mass, as discussed in Huang and Jin (2002). Note, however, that we have not used the MITgcm in a fully non-hydrostatic mode. The fully nonhydrostatic model involves an additional algorithmic step to compute the non-hydrostatic pressure contribution, which is complicated when using a nonlinear free surface. The changed algorithm represents a further perturbation and will surely lead to additional deviations from the solution of the hydrostatic model.

There is also convincing evidence that the changes due to Boussinesq effects are smaller than the errors introduced by other approximations and parameterizations generally made by ocean general circulation models.

Greatbatch et al. (2001) showed that the differences between a Boussinesq model and a non-Boussinesq model are reassuringly on the order of a few percent in the mean fields. Here we confirm that the differences between the nonBoussinesq pressure coordinate model and the Boussinesq height model are small in the time mean. But even the coarse resolution experiments are sensitive to small differences in the dynamics or parameterizations. These small perturbations lead to detectable changes in the computed circulation, particularly in the variability of bottom pressure and sea surface height.

It is particularly interesting to point out that all results shown here were obtained by using the same compiler on the same platform. Use of a different compiler or platform changes the results due to differing numerical floating point operations, and gives an additional clue about the order of magnitude of errors the Boussinesq approximations intro- duce: the numerical noise level. This conclusion is supported by the experiment that shows the differences due to round-off noise in the forcing fields. Such differences are as large as those due to Boussinesq effects.

McDougall et al. (2002) showed that the steady-state geostrophic Boussinesq equations are equivalent to the nonBoussinesq equations under the same dynamical regime. Therefore, at the coarse resolution used here, we can expect only small differences between a Boussinesq and a nonBoussinesq ocean model, because a good deal of the simulation should be geostrophically balanced. With increasing realism of the simulations (increased resolution, higher frequencies, etc.) that leads to sufficient ageostrophic and time-dependent motion one expects the Boussinesq effects to become more important. But at the same time, hydrostatic effects will become more prominent as well. Further, the model solutions of chaotic (eddy-resolving) systems will be highly sensitive to noise in the initial fields and the boundary conditions. Whether the Boussinesq effects will be discernible under these circumstances, remains questionable.

Still, models that do not make the Boussinesq approximation are recommended over the conventional height coordinate models when they are not more expensive to integrate. This point of view is taken for the puristic reason of abandoning an approximation. If, however, there is a considerable additional effort involved in integrating these mass conserving models, the minor effects of the Boussinesq approximation do not justify that cost. Instead, efforts to improve the parameterization of unresolved physics such as mixing will be more beneficial to OGCM solutions that relaxing the Boussinesq approximations.

One of the conclusions of this study is only indirectly related to the Boussinesq approximations. In the configuration of this study with mixed boundary conditions for temperature and salinity, the OGCM yields robust results on the large scale. But at the same time, it is so sensitive to small, in fact, almost indetectable changes in the parameterizations, largely unknown forcing fields, or approximations made, that one cannot expect two models that use a different coordinate system to stay on almost identical trajectories for an infinite time (of integration). The results presented here possibly depend on this detail. It is particularly important to compare the model variances while the two models are on a similar trajectory. Once the models have diverged, there is little hope of recovering the results shown.

When the property of exact mass conservation is required for a particular study, non-Boussinesq models must be preferred. However, current Boussinesq models in height coordinates still have their benefits. For instance, at eddy resolving scales, non-hydrostatic effects are believed to be important, and a non-hydrostatic model is much easier to formulate in height coordinates than in pressure coordinates. (On this note, the approach by Greatbatch et al. (2001) may allow the inclusion of non-Boussinesq effects into the non-hydrostatic version of the MITgcm and make the comparison of the different height coordinate models simpler. But we suspect that 
the Greatbatch et al. approach also introduces artificial differences due to the different structure of the equations (new terms) while the height-pressure isomorphic equations have the same structure.) Also, the representation of atmospheric pressure load in a pressure coordinate model is slightly more complicated than in a height coordinate model, because it involves two moving surfaces. Further, while the representation of bottom pressure in the pressure coordinate model is more natural, the bottom pressure diagnosed from the height coordinate model appears accurate enough in the light of other approximations made, namely the hydrostatic approximation.

Constraining pressure coordinate OGCMs with bottom pressure data is more straightforward in the same way that height coordinate models are more easily constrained by sea surface elevation data. Although we found high sensitivities to dynamical formulation under mixed boundary conditions, constrained models may show less sensitivity, as was the case with restoring boundary conditions. If this is the case then concerns about assimilation of bottom pressure even in height coordinate OGCMs are unwarranted, particularly since we have demonstrated that the evolution of net ocean mass can be quite accurately accounted for in such a model.

Acknowledgments. The authors thank Carl Wunsch and John Marshall for initiating this work and for many valuable comments. Raffaele Ferrari helped improve the manuscript. We also wish to thank Steve Griffies and one anonymous reviewer for their helpful comments. This work is a contribution to the ECCO (Estimating the Circulation and Climate of the Ocean) project. ML was funded JPL/NASA Contract \# 1205624. AA was funded by ONR/NOPP N00011-99-11050. JMC was funded by the MIT Climate Modeling Initiative.

APPENDIX

\section{A. Isomorphic Pressure and Height Coordinate Formulation of the Primitive Equations}

To familiarize the reader with an ocean model in pressure coordinates, the symmetry between the pressure and the height model formulations as pointed out by de Szoeke and Samelson (2002) and Marshall et al. (2003) is reviewed.

\section{A1. Boussinesq Hydrostatic Ocean Model in Height Coordinates}

The equations of motion in $z$ coordinates after making the Boussinesq approximation can be cast as

$$
\begin{aligned}
\frac{D \mathbf{u}}{D t} & =-\nabla_{z} \frac{p}{\rho_{0}}-f \mathbf{k} \times \mathbf{u}+\mathbf{F}, \\
\frac{\partial p}{\partial z} & =-g \rho, \\
\nabla_{z} \cdot \mathbf{u}+\frac{\partial w}{\partial z} & =0, \\
\frac{D \theta}{D t} & =Q, \\
\frac{D S}{D t} & =Q_{S} .
\end{aligned}
$$

In these equations, $\mathbf{u}$ is the horizontal velocity and $w$ the vertical velocity, $p$ is the full hydrostatic pressure, $\mathbf{F}$ is the frictional force. $Q$ and $Q_{S}$ represent the source terms of potential temperature $\theta$ and salinity $S$, for example, atmospheric fluxes of heat and freshwater, respectively. The subscript of $\nabla_{z}$ indicates that the gradient is taken along surfaces of constant $z$; the substantial time-derivative (rate-of-change) operator is

$$
\frac{D}{D t}=\left(\frac{\partial}{\partial t}\right)_{z}+\mathbf{u} \cdot \nabla_{z}+w \frac{\partial}{\partial z},
$$

where again the subscript at the differential operator means that these operators are applied at constant $z$. At the free surface, $z=\eta(x, y, t)$, we assume that the pressure is constant (taken to be zero) which leads to the boundary conditions

$$
\begin{aligned}
& p=0, \quad w=\frac{D \eta}{D t}-(P-E) \quad \text { at } z=\eta, \\
& w=-\mathbf{u} \cdot \nabla_{z} H \quad \text { at } z=-H(x, y) .
\end{aligned}
$$

$(P-E)$ is the freshwater flux into the ocean (precipitation minus evaporation).

A prognostic equation for the sea surface elevation $\eta$ is obtained by integrating (A3) and applying (A7) and (A8):

$$
\nabla_{z} \cdot\left(\int_{-H}^{\eta} \mathbf{u} d z\right)+\frac{\partial \eta}{\partial t}=(P-E) .
$$

Linearizing this equation is equivalent to neglecting a term $\nabla_{z} \cdot(\mathbf{u} \eta)$ in the surface boundary condition for $w$ (Roullet and Madec 2000).

\section{A2. Non-Boussinesq Hydrostatic Ocean Model in Pressure Coordinates}

The equations of motion written in terms of pressure as an independent variable are well-known and much used in dynamical meteorology (e.g., Haltiner and Williams 1980). Using an isomorphism (Marshall et al. 2003), the MITgcm 
implements these equations in pressure coordinates:

$$
\begin{aligned}
\frac{D \mathbf{u}}{D t} & =-\nabla_{p} \Phi-f \mathbf{k} \times \mathbf{u}+\mathbf{F}, \\
\frac{\partial \Phi}{\partial p} & =-\alpha, \\
\nabla_{p} \cdot \mathbf{u}+\frac{\partial \omega}{\partial p} & =0, \\
\frac{D \theta}{D t} & =Q, \\
\frac{D S}{D t} & =Q_{S} .
\end{aligned}
$$

Here, $\Phi=g z$ is the geopotential and $\nabla_{p}$ is the gradient along pressure surfaces; the substantial time-derivative (rateof-change) operator is

$$
\begin{aligned}
\frac{D}{D t} & =\left(\frac{\partial}{\partial t}\right)_{p}+\mathbf{u} \cdot \nabla_{p}+\omega \frac{\partial}{\partial p} \\
& =\left(\frac{\partial}{\partial t}\right)_{z}+\mathbf{u} \cdot \nabla_{z}+w \frac{\partial}{\partial z},
\end{aligned}
$$

where the pseudo velocity $\omega$, the pressure tendency, is defined by

$$
\omega=\frac{D p}{D t} .
$$

The specific volume $\alpha$ is given by the equation of state

$$
\rho^{-1}=\alpha=\alpha(S, \theta, p) \text {. }
$$

At this point, it is interesting to point out that the form of the continuity equation (A12), albeit resembling the incompressibility statement of Boussinesq models in $\mathrm{z}-$ coordinates, is a consequence of the hydrostatic assumption (A11) and does not require the neglect of dilatation $\rho^{-1} D \rho / D t$ (de Szoeke and Samelson 2002). Hence, a nonBoussinesq model with continuity equation(A12) conserves mass in contrast to a Boussinesq model, which conserves volume.

As before, we assume that the pressure is constant at the upper surface (taken to be zero). Any freshwater flux into the ocean (precipitation minus evaporation) appears as a boundary condition for $\omega$ (see Appendix B). The flux is scaled by the gravitational acceleration $g$ and the density of freshwater $\rho_{F W}=999.8 \mathrm{~kg} \mathrm{~m}^{-3}$ :

$$
\omega=g \rho_{F W}(P-E) \quad \text { at } p=0 .
$$

At the ocean bottom, $z=-H(x, y)$, the boundary condition is expressed in terms of bottom pressure $p_{b}(x, y, t)$ as

$$
\omega=\frac{\partial p_{b}}{\partial t}+\mathbf{u} \cdot \nabla_{p} p_{b} \quad \text { at } p=p_{b} .
$$

Together with the continuity equation (A12), the boundary conditions yield a prognostic equation for the bottom pressure

$$
\nabla_{p} \cdot\left(\int_{0}^{p_{b}} \mathbf{u} d p\right)+\frac{\partial p_{b}}{\partial t}=g \rho_{F W}(P-E),
$$

which is the analogue to equation(A9). Equation(A20) can be viewed as a statement of mass conservation for the entire water column.

\section{A3. Symmetry of the Primitive Equations and the Isomorphism in the MITgcm}

The two sets of equations in the preceding two sections have a strikingly similar form. By making the following substitutions, they can be transformed into each other:

$$
\begin{aligned}
z & \longleftrightarrow p, \\
w & \longleftrightarrow \omega, \\
p / \rho_{0} & \longleftrightarrow \Phi, \\
g \rho & \longleftrightarrow \alpha .
\end{aligned}
$$

In the model code of the MITgcm, these variables are replaced by general variables. A run time switch then selects the meaning they have in the present experiment. This makes it possible to use the identical dynamical kernel for simulations in both pressure and height coordinates (Marshall et al. 2003).

Exploiting this symmetry, both models can be summarized in terms of more general $r$ coordinates:

$$
\begin{aligned}
\frac{D \mathbf{u}}{D t} & =-\nabla_{r} \phi-f \mathbf{k} \times \mathbf{u}+\mathbf{F}, \\
\frac{\partial \phi}{\partial r} & =b \\
\nabla_{r} \cdot \mathbf{u}+\frac{\partial \dot{r}}{\partial r} & =0 \\
\frac{D \theta}{D t} & =Q \\
\frac{D S}{D t} & =Q_{S} .
\end{aligned}
$$

The general coordinate $r$ replaces $p$ and $z$, the vertical velocities $\omega$ and $w$ become $\dot{r}=D r / D t$, the general potential $\phi$ takes the place of $\Phi$ and $p / \rho_{0}$, and the generalized buoyancy $b$ is substituted for the specific volume $-\alpha$ and the scaled density $-g \rho / \rho_{0}$. The substantial time-derivative (rate-of-change) operator in this formulation is

$$
\frac{D}{D t}=\left(\frac{\partial}{\partial t}\right)_{r}+\mathbf{u} \cdot \nabla_{r}+\dot{r} \frac{\partial}{\partial r},
$$

where now the horizontal gradients are taken along the surfaces of constant $r$.

To see the symmetry in the kinematic boundary conditions it is convenient to distinguish between a (moving) free surface, which is at the top in height coordinates and at the ocean floor in pressure coordinates, and a fixed boundary surface, which conversely is at the top for pressure coordinates and at the bottom for height coordinates. The boundary conditions at the free and at the fixed boundary surfaces are

$$
\begin{array}{ll}
\dot{r}=\frac{D r_{s}}{D t} & \text { at } r=r_{s}, \\
\dot{r}=-\mathbf{u} \cdot \nabla_{r} R_{\text {fixed }} & \text { at } r=-R_{\text {fixed }}(x, y) .
\end{array}
$$


where $r=r_{s}$ is the free surface; $r_{s}$ is the bottom pressure $p_{b}$ in pressure coordinates and the sea surface elevation $\eta$ in height coordinates. $r=-R_{\text {fixed }}$ is the topography of the fixed surface, that is, 0 in pressure coordinates and depth $H$ in height coordinates. So with the additional substitutions for the boundary conditions

$$
\begin{aligned}
r_{s}: \quad \eta \longleftrightarrow p_{b}, \\
R_{\text {fixed }}: \quad H \longleftrightarrow 0
\end{aligned}
$$

the symmetry is complete, except for freshwater flux at the ocean surface. The flux $-\gamma(P-E)$ has to be added to the boundary condition (A28) or (A29), whichever is the one at the ocean-atmosphere interface. The scale factor $\gamma$ converts the freshwater flux into pressure or height units, that is, mass flux $\left(\gamma=g \rho_{F W}\right)$ or volume flux $(\gamma=-1)$, respectively. Finally, the prognostic equation for the free surface $r_{s}$ is again the same for both coordinate systems:

$$
\nabla_{r} \cdot\left(\int_{-R_{\mathrm{fixed}}}^{r_{s}} \mathbf{u} d z\right)+\frac{\partial r_{s}}{\partial t}=\gamma(P-E)
$$

\section{B. Natural Boundary Conditions in Pressure Coordinates}

At the surface, a freshwater flux not only dilutes the salinity, but adds mass to the water column. To represent this flux through the material surface (interface between atmosphere and ocean), the boundary condition for $\omega$ in pressure coordinates (or $w$ in height coordinates) has to be modified. Without a freshwater flux, $\omega=0$ at the surface. Including the flux leads to the boundary condition(A18), which may appear inconsistent at first, because the surface $p=0$ is a coordinate plane. Integrating the non-Boussinesq, hydrostatic continuity equation in height coordinates for $w$ over the top pressure layer with thickness $\Delta p$ yields

$$
0=\int_{\Delta p}^{0}\left\{\frac{\partial \rho}{\partial t}+\nabla_{z}(\rho \mathbf{u})+\frac{\partial}{\partial z}(\rho w)\right\} d p
$$

or

$$
0=\int_{-z_{1}(p=\Delta p)}^{\eta(p=0)}\left\{\frac{\partial \rho}{\partial t}+\nabla_{z}(\rho \mathbf{u})+\frac{\partial}{\partial z}(\rho w)\right\} d z
$$

$\eta$ and $-z_{1}$ are the respective heights of the surface pressure and the base of the layer. Multiplying through with $g$, using Leibniz's rule, and using the boundary condition(A7) for $w$, we get

$$
\begin{aligned}
0= & \frac{\partial}{\partial t} \Delta p+\nabla \cdot \int_{-z_{1}}^{\eta}(g \rho \mathbf{u}) d z \\
& -g \rho \frac{\partial \eta}{\partial t}-g \rho \mathbf{u} \cdot \nabla_{p} \eta \\
& +g \rho \frac{\partial\left(-z_{1}\right)}{\partial t}+g \rho \mathbf{u} \cdot \nabla_{p}\left(-z_{1}\right) \\
& +g \rho \frac{D \eta}{D t}-g \rho_{F W}(P-E)-\left.(g \rho w)\right|_{-z_{1}} .
\end{aligned}
$$

$\nabla_{p} \eta$ and $\nabla_{p} z_{1}$ are gradients along surfaces of constant pressure. With the layer-averaged velocity $\overline{\mathbf{u}}$ and observing that $\Delta p$ is constant $(\partial \Delta p / \partial t=0)$

$$
\begin{aligned}
0= & \nabla \cdot(\Delta p \overline{\mathbf{u}})-g \rho_{F W}(P-E) \\
& -\left.\left\{g \rho\left(w-\frac{\partial z_{1}}{\partial t}-\mathbf{u} \cdot \nabla_{p} z_{1}\right)\right\}\right|_{-z_{1}}
\end{aligned}
$$

or

$$
0=\nabla \cdot \overline{\mathbf{u}}+\frac{1}{\Delta p}\left(-g \rho_{F W}(P-E)+\left.\omega\right|_{-z_{1}}\right),
$$

because

$$
w=\frac{D z}{D t}=\frac{\partial z}{\partial t}+\mathbf{u} \cdot \nabla_{p} z+\omega \frac{\partial z}{\partial p} .
$$

Equation(B5) is the full continuity equation in pressure coordinates for the top layer. For $\Delta p \rightarrow 0$, one recovers the differential form, and the implied boundary condition for $\omega$ becomes

$$
\omega=g \rho_{F W}(P-E) \quad \text { at } p=0 .
$$

\section{Finite Volume Discretization of the Hydrostatic Equation}

Let $k$ be the index of the grid point at the center between the vertical cell interfaces $k-\frac{1}{2}$ and $k+\frac{1}{2}$. If the distance between these vertical cell interfaces is called $\Delta r_{k}$, a finite difference discretization of the hydrostatic equation (A23) that yields the potential $\phi$ at the center between two grid planes is

$$
\phi_{k+1}=\phi_{k}+\left(\frac{\Delta r_{k}+\Delta r_{k+1}}{2}\right) \cdot\left(\frac{b_{k}+b_{k+1}}{2}\right) .
$$

This formulation has been shown to conserve energy (Adcroft et al. 1997). However, an arbitrary choice about discretization at the top and bottom boundaries leaves the definition of bottom pressure (surface geopotential) somewhat ambiguous. This ambiguity can be avoided by using the finite volume discretization where the hydrostatic pressure is integrated over half levels as follows:

$$
\phi_{k+\frac{1}{2}}=\phi_{k-\frac{1}{2}}+\Delta r_{k} b_{k}
$$


and at the center points,

$$
\begin{aligned}
\phi_{k+1} & =\phi_{k+\frac{1}{2}}+\frac{1}{2} \Delta r_{k+1} b_{k+1} \\
& =\phi_{k}+\frac{1}{2}\left(\Delta r_{k} b_{k}+\Delta r_{k+1} b_{k+1}\right) .
\end{aligned}
$$

In this formulation, bottom pressure in the height coordinate model (or sea surface elevation in the pressure coordinate model) can be diagnosed consistently.

\section{Quasi-Hydrostatic Equations}

The momentum equations in spherical (height) coordinates are

$$
\begin{aligned}
\frac{D u}{D t}= & -\left\{\frac{u w}{R}-\frac{u v \tan \phi}{R}\right\} \\
& -\left\{\frac{2 \Omega w \cos \phi}{\partial}-2 \Omega v \sin \phi\right\} \\
& -\frac{\partial}{\partial x} \frac{p}{\rho_{0}}+F_{u} \\
\frac{D v}{D t}= & -\left\{\frac{v w}{R}-\frac{u^{2} \tan \phi}{R}\right\} \\
& -\{2 \Omega u \sin \phi\} \\
& -\frac{\partial}{\partial y} \frac{p}{\rho_{0}}+F_{v} \\
\frac{D w}{D t}= & -\left\{\frac{u^{2}+v^{2}}{R}\right\}-\{\underline{2 \Omega u \cos \phi}\} \\
\hline \underline{R} & -\frac{1}{\rho_{0}}\left(g \rho+\frac{\partial p}{\partial z}\right)+\underline{\underline{F_{w}}}
\end{aligned}
$$

where $D / D t=\partial / \partial t+\mathbf{v} \cdot \nabla$. Here, $\mathbf{v}=(u, v, w)$ is the three dimensional velocity and $\nabla=\left(\frac{1}{R \cos \phi} \frac{\partial}{\partial \lambda}, \frac{1}{R} \frac{\partial}{\partial \phi}, \frac{\partial}{\partial z}\right)$ is the three dimensional gradient operator in spherical coordinates. $\Omega$ is the frequency of earth rotation, $R$ the radius of the earth, and $\phi$ is the latitude. $F_{u, v, w}$ stand for all friction terms. The so-called metric terms are the terms in the first bracket after the equal sign in all equations, the Coriolis terms are in the second bracket after the equal signs. In the quasi-hydrostatic approximation the terms that are underlined twice are dropped. Making the full hydrostatic approximation amounts to dropping the total time derivative and the friction term $F_{w}$ in the vertical momentum equation and all terms involving $w$ or $\cos \phi$ in the horizontal equations, that is, all terms that are underlined once or twice (Marshall et al. 1997b). Note that for the quasi-hydrostatic approximations the classical hydrostatic equation is augmented by a metric term and a Coriolis term.

\section{REFERENCES}

Adcroft, A., C. Hill, and J. Marshall, 1997: Representation of topography by shaved cells in a height coordinate ocean model. Mon. Wea. Rev., 125(9), 2293-2315.

Balmino, G., F. Perosanz, R. Rummel, N. Sneeuw, H. Sünkel, and P. Woodworth, 1998: European views on dedicated gravity field missions: GRACE and GOCE, An Earth Sciences Division Consultation Document, ESA, ESD-MAG-REP-CON-001.

Brugge, R., H. L. Jones, and J. C. Marshall, 1991: Non-hydrostatic ocean modeling for studies of open-ocean deep convection. Deep Convection and Deep Water Formation in the Ocean, P. C. Chu and J. C. Gascard, Eds., vol. 57 of Elsevier Oceanogr. Ser., Elsevier Sci., pp. 325-340.

Campin, J.-M., A. Adcroft, C. Hill, and J. Marshall, 2003: Conservation of properties in a free-surface model. Ocean Modelling. In Press.

de Szoeke, R. A., and R. M. Samelson, 2002: The duality between the Boussinesq and Non-Boussinesq hydrostatic equations of motion. J. Phys. Oceanogr., 32(8), 2194-2203.

Dewar, W. K., Y. Hsueh, T. J. McDougall, and D. Yuan, 1998: Calculation of pressure in ocean simulations. J. Phys. Oceanogr., 28(4), 577-588.

Gent, P. R., and J. C. McWilliams, 1990: Isopycnal mixing in ocean circulation models. J. Phys. Oceanogr., 20(1), 150-155.

Greatbatch, R. J., 1994: A note on the representation of steric sea level in models that conserve volume rather than mass. $J$. Geophys. Res., 99(C6), 12,767-12,771.

Greatbatch, R. J., Y. Lu, and Y. Cai, 2001: Relaxing the Boussinesq approximation in ocean circulation models. J. Atmos. Ocean. Technol., 18(11), 1911-1923.

Griffies, S. M., R. C. Pacanowski, M. Schmidt, and V. Balaji, 2001: Tracer conservation with an explicit free surface method for $\mathrm{z}$ coordinate models. Mon. Wea. Rev., 129(5), 1081-1098.

Haltiner, G. J., and R. T. Williams, 1980: Numerical Prediction and Dynamical Meteorology. Wiley, 477 pp., 2nd edition.

Huang, R. X., and X. Jin, 2002: Sea surface elevation and bottom pressure anomalies due to thermohaline forcing. Part I: Isolated perturbations. J. Phys. Oceanogr., 32(7), 2131-2150.

Huang, R. X., X. Jin, and X. Zhang, 2001: An oceanic general circulation model in pressure coordinates. Adv. Atmos. Sci., 18(1), $1-22$.

Jackett, D. R., and T. J. McDougall, 1995: Minimal adjustment of hydrographic profiles to achieve static stability. J. Atmos. Ocean. Technol., 12(4), 381-389.

Jiang, S., P. H. Stone, and P. Malanotte-Rizzoli, 1999: An assessment of the Geophysical Fluid Dynamics Laboratory ocean model with coarse resolution: Annual-mean climatology. $J$. Geophys. Res., 104(C11), 25,623-25,645.

Levitus, S., and T. Boyer, 1994: World Ocean Atlas 1994. Volume 4: Temperature. NOAA, NOAA Atlas NEDSIS 4.

Levitus, S., R. Burgett, and T. Boyer, 1994: World Ocean Atlas 1994. Volume 3: Salinity. NOAA, NOAA Atlas NEDSIS 3.

Lu, Y., 2001: Including non-Boussinesq effects in Boussinesq ocean circulation models. J. Phys. Oceanogr., 31(6), 16161622 .

Marshall, J., A. Adcroft, C. Hill, L. Perelman, and C. Heisey, 1997a: A finite-volume, incompressible Navier Stokes model for studies of the ocean on parallel computers. J. Geophys. Res., 102(C3), 5753-5766.

Marshall, J., C. Hill, L. Perelman, and A. Adcroft, 1997b: Hydrostatic, quasi-hydrostatic, and nonhydrostatic ocean modeling. $J$. Geophys. Res., 102(C3), 5733-5752.

Marshall, J., A. Adcroft, J.-M. Campin, and C. Hill, 2003: Atmosphere-ocean modeling exploiting fluid isomorphisms. Submitted. 
McDougall, T. J., R. J. Greatbatch, and Y. Lu, 2002: On conservation equations in oceanography: How accurate are Boussinesq ocean models. J. Phys. Oceanogr., 32(5), 1574-1584.

McDougall, T. J., D. R. Jackett, D. G. Wright, and R. Feistel, 2003: Accurate and computationally efficient algorithms for potential temperature and density of seawater. J. Atmos. Ocean. Technol.. In press.

NOAA, 1988: Digital relief of the surface of the Earth, National Geophysical Data Center, Data Announcement 88-MGG02, NOAA, National Geophysical Data Center, Boulder, CO.

Power, S. B., and R. Kleeman, 1994: Surface heat flux parameterization and the response of ocean general circulation models to high-latitude freshening. Tellus, A 46(1), 8695.doi:10.1034/j.1600-0870.1994.00,008.x.

Rahmstorf, S., 1996: Comments on "Instability of the thermohaline circulation with respect to mixed boundary conditions: Is it really a problem for realistic models?". J. Phys. Oceanogr. 26(6), 1099-1105.

Roullet, G., and G. Madec, 2000: Salt conservation, free surface, and varying levels: A new formulation for ocean general circulation models. J. Geophys. Res., 105(C10), 23,927-23,942.

Spiegel, E. A., and G. Veronis, 1960: On the Boussinesq approximation for a compressible fluid. Astrophys. J., 131, 442-447.

Trenberth, K. M., J. G. Olson, and W. G. Large, 1990: The mean annual cycle in global ocean wind stress. J. Phys. Oceanogr., 20, 1742-1760.

Wunsch, C., and D. Stammer, 1998: Satellite altimetry, the marine geoid, and the oceanic general circulation. Ann. Rev. Earth and Planet. Sci., 26, 219-253.

Printed April 15, 2003. 